\title{
Intrusions detection using optimized support vector machine
}

\author{
Mehdi Moukhafi, Khalid El Yassini, Bri Seddik \\ Department of Mathematics and Computer Sciences, Faculty of Sciences, Moulay Ismail University, Morocco
}

\begin{tabular}{|c|c|}
\hline Article Info & ABSTRACT \\
\hline Article history: & Computer network technologies are evolving fast and the development of \\
\hline Received May 3, 2019 & $\begin{array}{l}\text { internet technology is more quickly, people more aware of the importance of } \\
\text { the network security. Network security is main issue of computing because }\end{array}$ \\
\hline Revised Oct 20, 2019 & the number attacks are continuously increasing. For these reasons, intrusion \\
\hline Accepted Jan 15, 2020 & $\begin{array}{l}\text { detection systems (IDSs) have emerged as a group of methods that combats } \\
\text { the unauthorized use of a network's resources. Recent advances in }\end{array}$ \\
\hline Keywords: & $\begin{array}{l}\text { information technology, specially in data mining, have produced a wide } \\
\text { variety of machine learning methods, which can be integrated into an IDS. }\end{array}$ \\
\hline $\begin{array}{l}\text { Genetic algorithm } \\
\text { Intrusion detection system }\end{array}$ & $\begin{array}{l}\text { This study proposes a new method of intrusion detection that uses support } \\
\text { vector machine optimizing optimizing by a genetic algorithm. to improve } \\
\text { the efficiency of detecting known and unknown attacks, we used a Particle }\end{array}$ \\
\hline $\begin{array}{l}\text { kdd99 } \\
\text { Particle swarm optimization }\end{array}$ & $\begin{array}{l}\text { Swarm Optimization algorithm to select the most influential features for } \\
\text { learning the classification model. }\end{array}$ \\
\hline
\end{tabular}

This is an open access article under the CC BY-SA license.

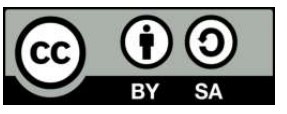

\section{Corresponding Author:}

Mehdi Moukhafi,

Informatics and Applications Laboratory (IA),

Department of Mathematics and Computer Sciences, Faculty of Sciences,

Moulay Ismail University,

Meknes, Morocco.

Email: mehdi.moukhafi@gmail.com

\section{INTRODUCTION}

Due to the tremendous growth of the Internet and Network based services, the severity o f network based computer attacks have significantly increased. Thus, an intrusion detection system (IDS) play a vital role in network security. Intrusion detection system tries to detect computer attacks by examining various data records. the IDS was presented for the first time by Anderson in 1980 [1], and later formalized by Denning [2], can be used in the global security politics, which includes other protection tools, such as firewalls and anti-virus software; Thus, it is important to take the advantage of these tools collaboration and complementarity. Actual IDS's based on heuristic rules, such as Snort are signature based system. The problem with such a system is that it cannot detect novel attacks whose signature is not available and hence generates a high rate of alerts. where constantly the environments were changing, the major drawback of approaches based signature is that they only detect known attacks, which implies a frequent updating of the rules database and the time for the implement. To overcome the mentioned problem above, many data mining techniques have been developed [3].

The data mining techniques are better applied equally for an anomaly intrusions detection, also for a knowledge-based intrusions detection [4]. The statistical analysis of the normal system behavior is one of the first approaches to intrusion detection. The statistics are used mathematically to describe an observed mechanism. Generally, the observations allow to get a rough description. For this, the value of certain observations is considered random variables. For each of its comments, a statistical model is used to describe the set of the corresponding random variable distributions. 
Learning algorithms can play an important role in detecting attacks (known or unknown). Additionally, the IDS's performances is considerably improved at the network level. SVM obtains a good detection performance in terms of classifying the flow of a network into normal or abnormal behaviors. Feng et al. [5] introduced an approach combining SVM with self-organized ant colony network. Kuang [6] propose a solution based on a combination of the SVM model with kernel principal component analysis (KPCA) and genetic algorithm. KPCA was used to reduce the dimensions of feature vectors, whereas GA was employed to optimize the SVM parameters. Al-Yaseen et al. [7] Propose a solution based on hybrid SVM and Extreme Learning Machine model Learned with data set built by a modified K- means The modified K-means is used to build new small training datasets representing the entire original training dataset. The rest of this work is organized as follows: Section 1 describes the PSO, SVM and GA methods, section 2 proposed architecture, section 3 simulation of results and evaluation of the algorithm. Section 4 conclusion and future work.

\section{THE USED METHODS}

\subsection{Particle swarm optimization}

Particle Swarm Optimization (PSO) is a stochastic optimization method, for the nonlinear functions, inspired by the social behavior of insect colonies, bird flocks, fish schools and other animal societies, PSO was invented by Russell Eberhart and James Kennedy [8] in 1995. Originally, the two began developing software simulations birds flocking around food sources, later after realizing that their algorithm solve optimization problems, they present [9] a discrete binary PSO algorithm developed from the previous PSO and operating in continuous variables. PSO is an iterative algorithm to find the best solution based on a population composed of many particles. For example, a flock of birds (particles) encircling an area where they can feel a hidden source of food. Whoever the closest to food warn others birds to move toward its direction. If any of the other birds circling closer to the target more than the first, it warbles stronger and the others move towards him. This scheme continues until one of the birds find food. A particle (candidate solution) that may move to the optimal position by updating its position and its speed. The speed of movement of a particle can be updated by the weight of inertia, cognitive learning factor, and the values of social learning factors.

\subsection{Support vector machine}

Support Vector Machine (SVM) is one of the most popular supervised machine learning algorithms. This is a classification model by evaluating data and identify patterns that retains excellent long generalization capabilities with an integrated resistance to overtraining. This generalization is based on solid theoretical foundations introduced by Vapnik [10]. An SVM model as shown in Figure 1 is an illustration of examples of points in two-dimensional space, where instances of different groups are separated by an area called margin.

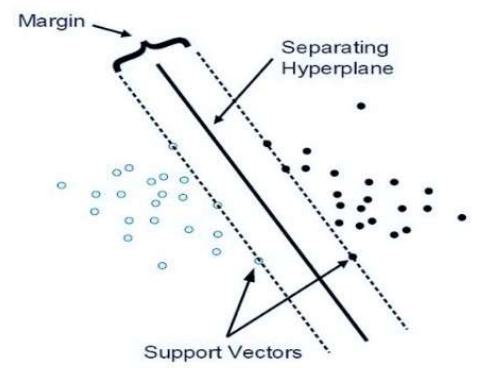

Figure 1. Classical example of SVM linear classifier

In the classification of support vector, the separation function is a linear combination of grains as given in (1) and are in contact with the support vector

$$
f(x)=\sum_{i \in S} \mu_{i} y_{i} x_{i}^{t} x+b
$$

where $\mu_{\mathrm{i}}$ is a Lagrange xi factor is training models, yi $\{+1,-1\}$ is the corresponding class labels and $\mathrm{S}$ denotes the set of support vectors.

Intrusions detection using optimized support vector machine (Mehdi Moukhafi) 


\subsection{Genetic algorithm}

Genetic Algorithm (GA) [11] a general adaptive optimization search methodology based on a direct analogy to Darwinian's principle of evolution and survival of fittest to optimize a population of candidate solutions towards a predefined fitness. The procedure for a genetic algorithm is:

- Initialization: Create an initial population. This population is usually randomly generated of $\mathrm{n}$ chromosomes (suitable solutions for the problem)

- Evaluation: Each chromosome $\mathrm{x}$ of the population is then evaluated and we calculate a 'fitness' for that individual.

- Selection: Select two parent chromosomes from a population, based on their fitness (the better fitness, the bigger chance to be selected)

- $\quad$ Crossover: create new individuals by combining aspects of our selected parent.

- Mutation: The algorithm creates mutation children by randomly changing the genes of individual parents. Mutation typically works by making very small changes at random to an individual genome.

- Test: If the end condition is satisfied, stop, and return the best solution in current population, else return to selection step.

\section{PROPOSED MODEL FOR INTRUSION DETECTION}

\subsection{PSO features selection}

From an artificial intelligence perspective, create a classifier means creating a template for data, or perfect for a model is to be as simple as possible. Reducing the number of parameters, then reduces the number of parameters necessary for the description of this model.

- It improves the classification performance: learning time, his speed and power of generalization.

- It increases the comprehensibility of data. This data selection is to select an optimum subset of relevant variables from a set of original variables.

The KDD99 has 41 variables, it is relatively a large number to be processed by the classifier, the latter in the learning phase cannot complete execution within a reasonable time, then the selection can reduce the feature space. We used PSO to select the optimum features, that have the most impact on the prediction of the model, so we reduce the number of fields from 41 to 16 Table 1.

Selected features:

$2,3,4,5,6,8,11,14,23,26,29,30,35,36,37,38$

Table 1. Comparison of number of features between the data set and subset

\begin{tabular}{cc}
\hline Data set & Subset \\
\hline $0,1,18,10,491,0,0,0,0,0,0,0,0,0,0,0,0,0,0,0,0,0,2,2$, & $1,18,10,491,0,0,0,0,2,0,1,0,0.03,0.17,0,0$, normal \\
$0,0,0,0,1,0,0,150,25,0.17,0.03,0.17,0,0,0,0.05,0$, normal & \\
\hline
\end{tabular}

\subsection{Architecture of proposed IDS}

To implement our proposed approach, the RBF kernel function is used for the SVM classifier because the RBF kernel function has an excellent performance for the management of higher-dimensional data and requires that only two parameters:

- C (penalty parameter): This parameter, common to all SVM kernels, trades off misclassification of training examples against simplicity of the decision surface.

- $\quad \gamma$ (gamma parameter): It is a specific parameter to RBF kernel function, gamma defines how much influence a single training example has.

The parameters ( $\mathrm{C}$ and $\mathrm{g}$ ) used as input attributes must be optimized using genetic algorithm. To precisely establish a GA-SVM based intrusion detection system, the following main steps (as shown in Figure 2) must be proceeded. The detailed explanation is as follows:

Step 1: Features selection using PSO for a training data set (kdd99_p)

Step 2: Initialization of population by a genetic algorithm

Step 3: Genetic algorithm process: In this step, the system searches for better solutions by genetic operations, including selection, crossover, mutation (Described in the previous session)

Step 4: Training SVM classifier using optimized parameters $(\mathrm{C}, \gamma)$

Int J Adv Appl Sci, Vol. 9, No. 1, March 2020: $62-66$ 
Step 5: Fitnessevaluation. For each chromosome representing $C, \gamma$, training dataset is used to train the SVM classifier, each chromosome is evaluated by fitness function

Step 6: Termination criteria :When the termination criteria are satisfied, we evaluate a model using a full kdd99 data set; otherwise, return to step 3.

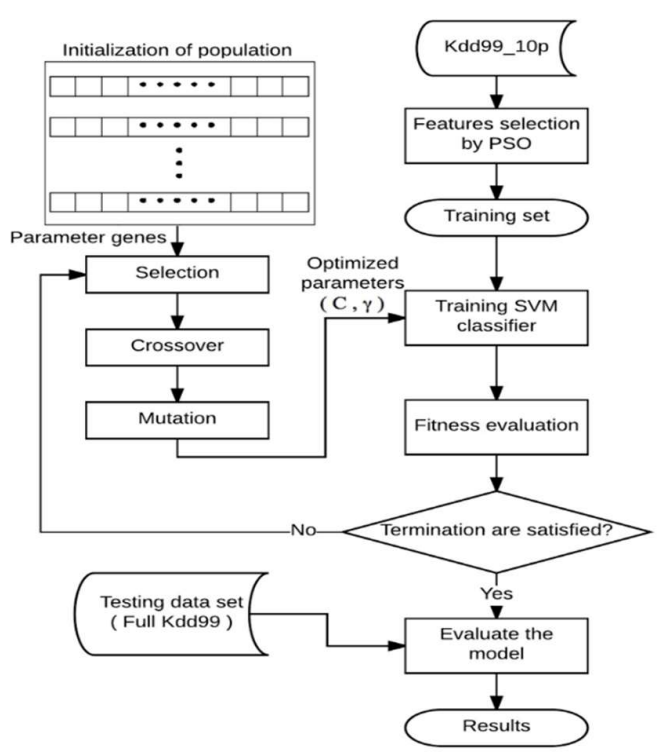

Figure 2. Proposed algorithm

\section{EXPERIMENT SETUP AND PERFORMANCE EVALUATION}

In this section, we evaluate the performance of the proposed model. All experiments were conducted on a calculation station $24 \mathrm{CPU}$ Intel Core $2.13 \mathrm{GHz}, 48 \mathrm{~GB}$ RAM, running under Linux CentOS 7. The implementation was coded using the Java language.

\subsection{Data set}

Cyber Systems and Technology Group of MIT Lincoln Laboratory [12] simulated LAN US Air Force LAN with multiple attacks and captured nine weeks TCPdump data. This database was first used for competitions kdd99, but since it has become the database test to the IDS's based on a behavioral approach. KDD Cup 1999 provided both the training dataset, it is called KDd99 10p.

Each connection record consists of approximately 100 bytes. This was converted into about $49 *$ 105 connection vectors each one contains 41 fields. This database is collected by simulating attacks on different platforms such as Windows, Unix, etc ... Four gigabytes of raw data compressed TCP dump is transformed into five million connections files. The attacks are divided into four main categories: Denial of Service Attack (DOS), Probing, User to Root Attack (U2R), Remote to Local Attack (R2L).

\subsection{Anomaly detection results}

This section describes the obtained results from the experiment by applying the proposed algorithms on the data set kdd99. The performance of the proposed method of intrusion detection was evaluated on all KDD99 data set, $10 \%$ of the KDD99 data set were used for training the GA-SVM model after the features selection by PSO. Tables 2 illustrates the confusion matrix. this system achieves a top performance of up to $96,01 \%$ with a reasonable false alarm rate of $0,02 \%$ and a detection rate of $96,38 \%$.

Table 2. Confusion matrix

\begin{tabular}{cccccc}
\hline \multirow{2}{*}{ Actual Class } & Normal & DOS & Probe & R2L & U2R \\
\hline Normal & 713680 & 106761 & 26767 & 30811 & 15762 \\
DOS & 580 & 3878986 & 3784 & 6 & 14 \\
Probe & 519 & 6646 & 33797 & 3 & 137 \\
R2L & 4 & 0 & 17 & 923 & 182 \\
U2R & 0 & 0 & 0 & 21 & 31 \\
\hline
\end{tabular}

Intrusions detection using optimized support vector machine (Mehdi Moukhafi) 
Figure 3 shows the detection rate classified by attack, the proposed algorithm has detected 99,89\% of DOS attacks whom are the most used by hackers. For Probe attacks a rate of $82,23 \%$ is correctly classified, $81,97 \%$ for R2L attacks and $59,62 \%$ for U2R, the low rate of detection U2R attacks can be explained by the insufficient number of data record-learning. To compare a detection rate, Figure 4 compares the proposed method with approaches that use only the entire KDD Cup 1999 dataset as a testing dataset because several researchers used only part of the KDD Cup 1999. The above results show that our approach enhances the performance of IDS. GA-SVM with PSO selection features is more reliable than state- ofthe-art methods. The strengths of the proposed method are the highly improved detection accuracy compared with other methods because of the high reduction of original training dataset size which simplified the learning phase of the classifier and the SVM parameters optimisation by GA.

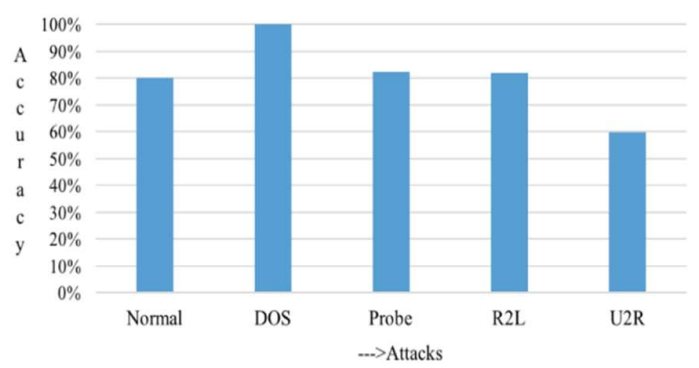

Figure 3. The accuracy rates per attack

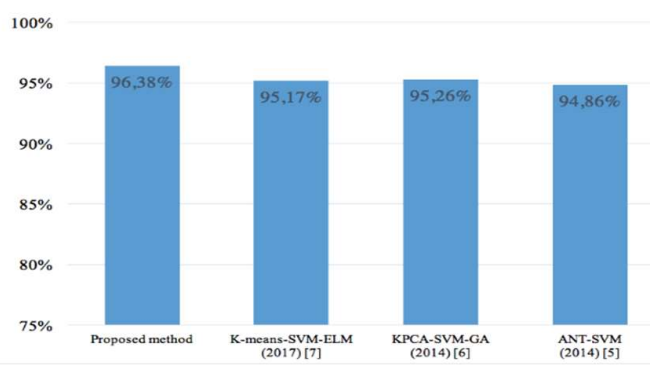

Figure 4. Comparison of proposed model with other methods by detection rate

\section{CONCLUSION}

In this paper, a Novel hybrid GA-SVM with PSO feature selection-based is proposed for intrusion detection. The proposed model is marked by a significantly better performance. RBF kernel function is used for improve the performance of SVM classification model, GA is used to select suitable parameters for SVM classifier and PSO is designated to select a features of the training dataset and provide new high-quality training datasets that can improve the overall performance of GA-SVM. We also carried out comparisons of our method against other methods, and have shown a noticeable performance. For future work, we want to develop more approaches to combine several machine learning techniques into one predictive model, using meta-algorithms, to increase the rate of detection of attacks.

\section{REFERENCES}

[1] J.P. Anderson, Computer security threat monitoring and surveillance. Technical Report, Fort Washington, PA, USA, 1980.

[2] D. E. Denning, "An Intrusion-Detection Model," IEEE Transactions on Software Engineering, Vol. 13, No. 2, pp. 222-232, 1987.

[3] S. Forrest, S.A. Hofmeyr, A. Somayaji, and T.A. Longstaff, "A sense of self for Unix processes," Proceedings of IEEE Symposium on Security and Privacy, Washington, pp. 120-128, 1996.

[4] W.L.W. Lee, SJ, and Mok KW., "A data mining framework for building intrusion detection models," Proceedings of the 1999 IEEE Symposium on Security and Privacy, California, pp. 120-132, 1999.

[5] W. Feng, Q. Zhang, G. Hu, and J.X. Huang, "Mining network data for intrusion detection through combining SVMs with ant colony networks," Future Generation Computer Systems, Vol. 37, pp. 127-140, 2014.

[6] F. Kuang, W. Xu, and S. Zhang, "A novel hybrid KPCA and SVM with GA model for intrusion detection," Applied Soft Computing Journal, Vol. 18, pp. 178-184, 2014.

[7] W.L. Al-Yaseen, Z.A. Othman, and M.Z.A. Nazri, "Multi-level hybrid support vector machine and extreme learning machine based on modified K-means for intrusion detection system," Expert Systems with Applications, Vol. 67, pp. 296-303, 2017.

[8] J. Kennedy and R. Eberhart, "Particle swarm optimization," Proceedings of IEEE International Conference, Vol. 4, pp. 1942-1948, 1995.

[9] J. Kennedy and R. Mendes, "Population structure and particle swarm performance," Proceedings of the 2002 Congress on Evolutionary Computation, Vol. 2, pp. 1671-1676, 2002.

[10] C. Cortes and V. Vapnik, "Support Vector Networks," Machine Learning, Vol. 20, No. 3, pp. 273-297, 1995.

[11] O. Kramer, Genetic Algorithm Essentials. Cham: Springer International Publishing, 2017.

[12] M. Tavallaee, E. Bagheri, W. Lu, and A.A. Ghorbani, "A detailed analysis of the KDD CUP 99 data set," in EEE Symposium on Computational Intelligence for Security and Defense Applications, pp. 1-6, 2009. 